\title{
Acute Glaucoma Filtering Surgery Failure following Herpes Zoster Ophthalmicus Infection
}

\author{
Stylianos A. Kandarakis Andreas Diagourtas Petros Petrou \\ Filippos Vingopoulos Konstantinos Droutsas \\ Evangelia Papakonstantinou lias Georgalas \\ 1st Opthhalmology Department, "G.Gennimatas" Hospital, National and Kapodistrian \\ University of Athens, Athens, Greece
}

\author{
Keywords \\ Glaucoma · Herpes zoster ophthalmicus - Trabeculectomy · Glaucoma filtering surgery
}

\begin{abstract}
Herein, we report a case of acute failure of a previously successful trabeculectomy, following an infection with herpes zoster ophthalmicus (HZO). HZO remains a common infection, especially among elderly and immunocompromised patients. There is a strong link between $\mathrm{HZO}$ infection, the incidence of secondary glaucoma, and the need for glaucoma filtering surgery. Though, to our knowledge, there are no cases reporting on the effect that a concomitant infection may have on a previously successful trabeculectomy. In our case, a 76-year-old immunocompetent male with primary open-angle glaucoma in both eyes and a history of a successful right eye trabeculectomy 1 year earlier presented with acute primary $\mathrm{HZO}$ involving the ophthalmic branch of the right trigeminal nerve. Appropriate topical and systemic treatment was immediately initiated. Three days later, the trabeculectomy bleb showed hyperemia and flattening and concomitant rise of intraocular pressure was noted. A week later, the cutaneous signs were improving yet the trabeculectomy had failed and high intraocular pressure was established, requiring both topical and systemic antiglaucoma medications. Our study suggests that a previously successful trabeculectomy may manifest signs of compromise and subsequent failure following a HZO infection.
\end{abstract}

(c) 2021 The Author(s).

Published by S. Karger AG, Basel

\section{Introduction}

Varicella-zoster virus is a neurotrophic alpha-herpesvirus that usually presents as a primary infection in childhood causing acute varicella infection, a disease also known as chicken pox. Reactivation in adult life can cause a vesicular rash known as shingles and may 
involve a single or multiple ganglia simultaneously. It is estimated that in the US 300,000500,000 , adults are affected by shingles every year and $17-25 \%$ of the cases exclusively involve the eye, an entity called herpes zoster ophthalmicus (HZO) [1]. HZO has a great extent of ocular involvement and the virus may be affecting almost any part of the eye by perineural and intraneural inflammation, including the eyelids, the conjunctiva, the sclera, the cornea, the trabeculum, the retina, and the optic nerve, while the reasons for the extent of the involvement remain unknown.

Primary open-angle glaucoma (POAG) is the most common progressive optic neuropathy leading to characteristic changes in the optic nerve head and ganglion cell layer loss. POAG holds one of the leading roles in worldwide blindness, and it is estimated that the incidence of the disease will increase in the following decades [2]. Although current surgical treatment for regulating the intraocular pressure is enriched with new ideas and new surgical devices, there is no significant doubt that trabeculectomy remains the gold standard surgical procedure, especially for advanced disease with significant visual field loss. In the last decade, by introduction and intraoperative administration of antimetabolites, antiogenic, and anti-inflammatory medication, we have increased both success rates and viability of the surgical outcome. Even though the success rate of trabeculectomy shows a great variability in literature, there is sufficient data that the presence of inflammation affects the surgical outcome and viability of the surgery as implied by the decreased success rate between patients with primary and secondary or refractory glaucomas [3].

There is a strong link between HZO infection and secondary glaucoma, with the incidence of secondary glaucoma varying between 16 and $56 \%$, mainly depending on the extent of the virus involvement to the ocular structures. In their article, Thean et al. [4] reported an incidence of glaucoma up to $56 \%$ in patients that have developed HZO from which, $26 \%$ required glaucoma filtering surgery to control the IOP. What has not been clearly stated or described thus far, is the effect that a latent infection could have on a patient that already has glaucoma filtering surgery and whether the inflammation caused by the virus flare could diminish the outflow capacity of the trabeculectomy. In this case report, we describe a case of a patient that had a successful trabeculectomy 1 year prior to HZO infection and developed an acute bleb failure and raise of IOP within 3 days from his initial presentation at our office, full, and appropriate medical treatment notwithstanding.

\section{Case Presentation}

A 76-year-old immunocompetent male called our office and complained about mild fatigue accompanied by a mild diffuse pain in the right side of his face. The patient was advised to come to the office, which he did 3 days later when a characteristic maculopapular rash, respecting the midline, had been established on the right side of his face, in the distribution of the ophthalmic branch of the trigeminal nerve. The patient was thoroughly examined and had only signs of blepharoconjunctivitis with eyelid swelling, presence of vesicular rash in both the upper and lower eyelid, and mild hyperemia of the conjunctiva, without fluorescein or rose Bengal staining. Hutchinson sign was absent, and no other pathological findings of the cornea or signs of intraocular involvement were noticed.

Thirteen months earlier, he had undergone a trabeculectomy procedure on his right eye for advanced glaucoma with inadequate IOP control despite being on the maximum topical medical treatment and acetazolamide $250 \mathrm{mg}$, three times a day. Based on the advanced stage of the disease with only central island of visual field remaining, a glaucoma filtering surgery was decided. Preoperative IOP was between 23 and $25 \mathrm{~mm} \mathrm{Hg}$.

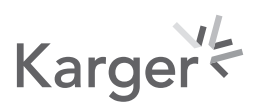


A trabeculectomy was performed with our standard technique with a square flap and two 10-0 nylon sutures at each corner. MMC $0.2 \mathrm{mg} / \mathrm{mL}$ was applied for $2.5 \mathrm{~min}$ intraoperatively and watertight closure was achieved. At 12 months postoperatively, the patient had an IOP between 8 and $10 \mathrm{~mm} \mathrm{Hg}$ on multiple visits with no antiglaucoma medications used, and hence, the trabeculectomy was considered successful. Patient's last follow-up visit was 10 days prior to the herpes zoster infection when the patient was examined and photos were taken (Fig. 1). The bleb was diffuse with mild vascularity, no signs of encapsulation and the Seidel test was negative. The bleb was staged according to the IBAGS (Indiana Bleb Appearance Grading Scale) as H2 $\backslash \mathrm{E} 1 \backslash \mathrm{V} 2 \backslash \mathrm{S} 0$ [5], IOP was measured with Goldmann applanation tonometry at $9 \mathrm{~mm} \mathrm{Hg}$, and surgery was documented still successful.

On current examination, the intraocular pressure was slightly more elevated than usual at $15 \mathrm{~mm} \mathrm{Hg}$ with only a mild hyperemia noted over the trabeculectomy bleb. The patient was diagnosed with a primary HZO infection and was started on valacyclovir 1,000 mg three times per day, prednisolone acetate $1 \%$ three times a day, and topical lubrication. On the third day, the bleb was noted to be flattered more hyperemic, and the IOP was not controlled, measured at $25 \mathrm{~mm} \mathrm{Hg}$. The cornea was not involved, and the anterior chamber had no inflammation. At that point, the patient was placed on topical glaucoma medication with b-blocker and carbonic anhydrase inhibitors, and previous treatment was continued. One week after the initial presentation, even though the cutaneous symptoms had improved, the bleb was completely flat and a very high IOP was established besides topical treatment with antiglaucoma drops at $35 \mathrm{~mm} \mathrm{Hg}$ (Fig. 2). At that point, a decision not to try needling with 5-FU injection was made because of the significant scarring and flattening of the bleb.

Six months after the infection, the patient had a failed bleb and required both topical maximal therapy with antiglaucoma medication and carbonic anhydrase inhibitors per os to control the IOP in his right eye. Secondary to the elevated IOP the patient's visual field deteriorated and the remaining central island constricted significantly.

\section{Discussion}

HZO remains a common ophthalmic infection mainly affecting elderly and immunocompromised patients. While the factors that determine the extent of ocular manifestations remain to be better understood, the complications produced by both viral replication and the accompanied inflammation may in some cases be vision threatening for some patients.

The association between HZO and secondary IOP elevation (16-56\%) has been described [6] and usually involves corneal and/or uveal manifestations of the viral infection. Approximately, $15 \%$ of these patients will require glaucoma filtering surgery to control the elevated IOP.

Fig. 1. Bleb morphology of the patient 10 days prior to HZO infection showing a posterior draining, elevated, mildly vascularized functioning bleb. HZO, herpes zoster ophthalmicus.

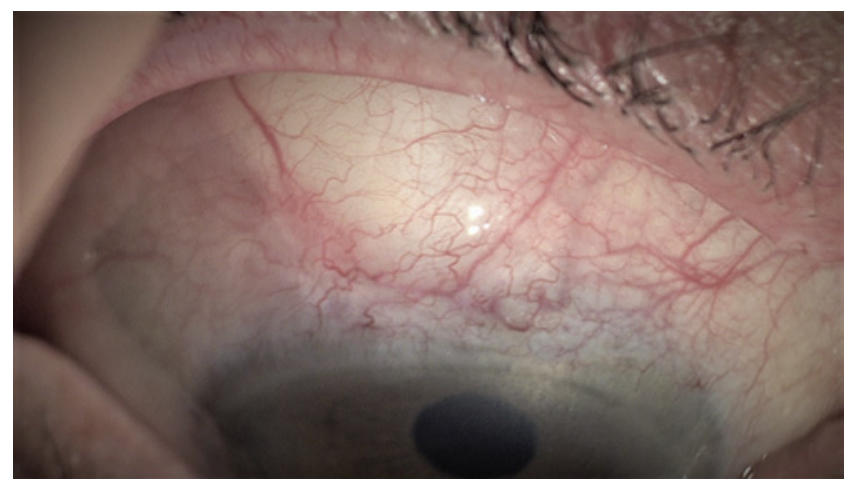


Fig. 2. Bleb morphology 1 week after HZO infection, revealing flattening and thinning of the bleb suggesting failure of glaucoma filtering surgery shortly after HZO infection. HZO, herpes zoster ophthalmicus.

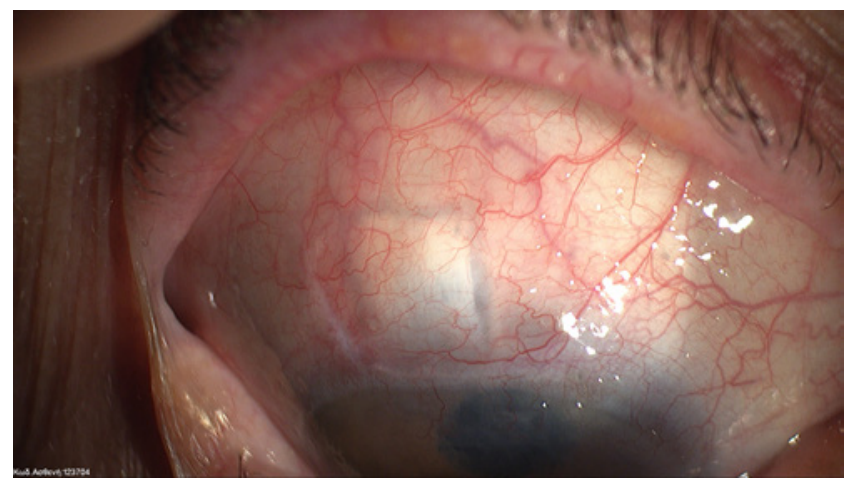

The success rate of glaucoma filtering surgery in these patients is lesser than in patients with POAG, and it is well recognized that the crucial factor for a successful outcome remains the adequate control of inflammation both pre- and postoperatively [7]. That observation verifies that glaucoma filtering surgery remains strongly related to the inflammatory status of the involved tissues including the conjunctiva and the underlying Tenon's capsule that have been found to be the birthplace of many inflammatory mediators [8].

Varicella virus has been shown to stimulate the production of many inflammatory mediators including IL-6 and TNF-a [9] the high presence of which has been recently related to compromised success of trabeculectomy $[8,10]$. To our knowledge, this is the first case of acute trabeculectomy failure secondary to an adequately treated HZO infection with both topical steroid treatment and systemic antiviral therapy with valacyclovir. Of note, no signs of scleritis, keratitis, or uveitis were present, yet the effect of the virus in the ocular tissue seemed to be substantial enough to lead to an acute trabeculectomy failure.

A possible explanation could be that a very high of varicella-zoster virus viral load led to cytosine release by both the conjunctiva and the Tenon's capsule as evident by involvement of the tissues on clinical exam. Even though the clinical signs of hyperemia of the bleb associated with the gradual elevation of IOP rendered the failing trabeculectomy apparent, the rapid course following the HZO infection is noticeable. Considering the management that was taken after the infection, controversial remains the use of steroids that could theoretically have contributed in further IOP elevation. That explanation seems unlikely since the patient had a successful trabeculectomy and the signs of acute inflammation are strongly related to glaucoma filtering surgery. It is also worth mentioning that a decision not to perform needling with 5-Fu injections was also taken. The decision was based on the amount of scarring, the rapid course, and the lack of knowledge on the effect that 5-Fu might have on herpes zoster virus even though there is strong suggestion in literature on the beneficial effect of $5-\mathrm{Fu}$ on postherpetic neuralgia [11].

\section{Conclusion}

Our study suggests that a previously successful trabeculectomy may manifest signs of compromise and subsequent failure following a HZO infection. In our case, this correlation seems to be causal since the trabeculectomy was stable and functional for an adequately long time to be considered successful, yet failed when an eye infection occurred. Further research with larger samples should be directed toward the pathophysiology of such a phenomenon in order to clarify whether such a correlation between a failed previously successful trabeculectomy and a concomitant eye infection is causal and identify measures of prevention.

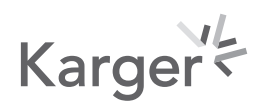


Kandarakis et al.: Acute Glaucoma Filtering Surgery Failure

\section{Clinical Significance}

This case should serve as an alarm for both glaucoma specialists as well as comprehensive ophthalmologists that a primary infection or possibly a flare of HZO infection involving the facial side of an eye having undergone a trabeculectomy carries a major threat for the function of glaucoma filtering surgery.

\section{Statement of Ethics}

Written informed consent was obtained from the patient for publication of this case report and any accompanying images.

\section{Conflict of Interest Statement}

The authors have no conflicts of interest to declare.

\section{Funding Sources}

The authors have no financial interest or funding to disclose.

\section{Author Contributions}

Stylianos A. Kandarakis: conception, data collection, manuscript drafting, Andreas Diagourtas: data collection and manuscript drafting, Petros Petrou: data analysis and manuscript drafting, Filippos Vingopoulos: manuscript drafting, Konstantinos Droutsas: manuscript drafting, Evangelia Papakonstantinou: manuscript drafting, Ilias Georgalas: critical revision and final approval.

\section{References}

1 Tran KD, Falcone MM, Choi DS, Goldhardt R, Karp CL, Davis JL, et al. Epidemiology of herpes zoster ophthalmicus: recurrence and chronicity. Ophthalmology. 2016;123(7):1469-75.

2 Gupta P, Zhao D, Guallar E, Ko F, Boland MV, Friedman DS. Prevalence of glaucoma in the United States: the 2005-2008 National Health and Nutrition Examination Survey. Invest Ophthalmol Vis Sci. 2016;57(6):290513.

3 Iwao K, Inatani M, Seto T, Takihara Y, Ogata-Iwao M, Okinami S, et al. Long-term outcomes and prognostic factors for trabeculectomy with mitomycin $\mathrm{C}$ in eyes with uveitic glaucoma: a retrospective cohort study. J Glaucoma. 2014;23(2):88-94.

4 Thean JH, Hall AJ, Stawell RJ. Uveitis in herpes zoster ophthalmicus. Clin Exp Ophthalmol. 2001;29(6):406-10.

5 Cantor LB, Mantravadi A, WuDunn D, Swamynathan K, Cortes A. Morphologic classification of filtering blebs after glaucoma filtration surgery: the Indiana Bleb Appearance Grading Scale. J Glaucoma. 2003;12(3):26671.

6 Panek WC, Holland GN, Lee DA, Christensen RE. Glaucoma in patients with uveitis. Br J Ophthalmol. 1990; 74(4):223-7.

7 Shimizu A, Maruyama K, Yokoyama Y, Tsuda S, Ryu M, Nakazawa T. Characteristics of uveitic glaucoma and evaluation of its surgical treatment. Clin Ophthalmol. 2014;8:2383-9.

8 Seong GJ, Hong S, Jung SA, Lee JJ, Lim E, Kim SJ, et al. TGF-beta-induced interleukin-6 participates in transdifferentiation of human Tenon's fibroblasts to myofibroblasts. Mol Vis. 2009;15:2123-8.

\section{Karger's}


9 Wang JP, Kurt-Jones EA, Shin OS, Manchak MD, Levin MJ, Finberg RW. Varicella-zoster virus activates inflammatory cytokines in human monocytes and macrophages via toll-like receptor 2. J Virol. 2005;79(20):1265866.

10 Cvenkel B, Kopitar AN, Ihan A. Inflammatory molecules in aqueous humour and on ocular surface and glaucoma surgery outcome. Mediators Inflamm. 2010;2010:939602.

11 Whitley RJ, Volpi A, McKendrick M, van Wijck A, Oaklander AL. Management of herpes zoster and post-herpetic neuralgia now and in the future. J Clin Virol. 2010;48(Suppl 1):S20-8. 